\title{
Practice effects in a longitudinal, multi-center Alzheimer's disease prevention clinical trial
}

\author{
Erin L Abner ${ }^{1}$, Brandon C Dennis ${ }^{1,2}$, Melissa J Mathews ${ }^{1,2}$, Marta S Mendiondo ${ }^{1,3}$, Allison Caban-Holt ${ }^{1,5}$, \\ Richard J Kryscio ${ }^{1,3,4}$, Frederick A Schmitt ${ }^{1,2,5,6,8^{*}}$ for the PREADViSE Investigators John J Crowley ${ }^{7}$ and for the \\ SELECT Investigators
}

\begin{abstract}
Background: Practice effects are a known threat to reliability and validity in clinical trials. Few studies have investigated the potential influence of practice on repeated screening measures in longitudinal clinical trials with a focus on dementia prevention. The current study investigates whether practice effects exist on a screening measure commonly used in aging research, the Memory Impairment Screen (MIS).

Methods: The PREADViSE trial is a clinical intervention study evaluating the efficacy of vitamin E and selenium for Alzheimer's disease prevention. Participants are screened annually for incident dementia with the MIS. Participants with baseline and three consecutive follow-ups who made less than a perfect score at one or more assessments were included in the current analyses $(\mathrm{N}=1,803)$. An additional subset of participants with four consecutive assessments but who received the same version of the MIS at baseline and first follow-up ( $N=301)$ was also assessed to determine the effects of alternate forms on mitigating practice. We hypothesized that despite efforts to mitigate practice effects with alternate versions, MIS scores would improve with repeated screening. Linear mixed models were used to estimate mean MIS scores over time.
\end{abstract}

Results: Among men with four visits and alternating MIS versions, although there is little evidence of a significant practice effect at the first follow-up, mean scores clearly improve at the second and third follow-ups for all but the oldest participants. Unlike those who received alternate versions, men given the same version at first follow-up show significant practice effects.

Conclusion: While increases in the overall means were small, they represent a significant number of men whose scores improved with repeated testing. Such improvements could bias case ascertainment if not taken into account.

Keywords: Practice effects, Clinical trials, Alzheimer's disease, Neuropsychological assessment

\section{Background}

Serial cognitive assessment is used in clinical practice, clinical trials, and longitudinal studies of aging and dementia to track cognitive fluctuations over time and to identify clinically significant declines in performance suggestive of mild cognitive impairment (MCI) or dementia. Screening measures with specific cut-points reflecting probable cognitive impairment are also frequently used as brief, first-line measures of gross cognitive functioning in

\footnotetext{
* Correspondence: fascom@uky.edu

${ }^{1}$ Sanders-Brown Center on Aging, University of Kentucky, Lexington, KY, USA ${ }^{2}$ Departments of Neurology, University of Kentucky Lexington, Kentucky, USA Full list of author information is available at the end of the article
}

both clinical and research settings. For example, patients performing below the cut-point on a screening measure may be referred for more extensive diagnostic evaluation. Research participants may be screened into or out of studies based upon whether their performance lies above or below the cut-point of the measure. When cognitive instruments are used repeatedly, it is imperative to know not only the sensitivity, specificity, positive predictive value, and negative predictive value of the instruments, but also their behavior over time.

Practice effects (PE) represent one aspect of that behavior. PE are distinct from random fluctuations in performance and refer to bias due to familiarity with test

\section{Biomed Central}


items and procedures when a test is retaken [1]. Longitudinal studies of cognitive aging are highly dependent on repeated testing with neuropsychological measures. For example, dementia prevention trials such as the Alzheimer's Disease Anti-inflammatory Prevention Trial (ADAPT) [2], Gingko Evaluation of Memory Study (GEMS) [3], and the Prevention of Alzheimer's Disease by Vitamin E and Selenium (PREADViSE) trial [4] rely heavily on repeated cognitive screening measures and standardized cognitive batteries for case ascertainment and tracking response to treatment.

Most studies demonstrating practice effects have involved test-retest paradigms over short time intervals [5-9] or have been conducted primarily with impaired populations [10-12]. Nonetheless, repeated testing effects have been well documented [13-17], and performance variability has been demonstrated to be influenced by age [18-21], fluid intelligence [21], clinical population [10,22], retest interval $[9,12,23,24]$, and the test or neurocognitive domain assessed [25,26]. Knowledge of the effects of repeated presentation is essential for interpretation of results. For example, PE can potentially alter the measure's sensitivity to cognitive change and have been found to account for between 31 and $83 \%$ of the variance in follow-up test scores [26]. Further, PE could influence dementia detection in prevention trials when screening measures are used, especially given known $\mathrm{PE}$, even for participants with Alzheimer's disease (AD), on measures such as the Mini-Mental State Exam [12].

Furthermore, PE may persist over long periods of time. In the UK, Rabbit and colleagues [20] examined PE over a 17 -year period in 5,899 participants, ages 49 to 92 . Similar to other studies, they found the greatest gain in performance between the first and second presentation but observed gains due to practice on intelligence tests over intervals of several years. In a separate sample studied over a 20-year period, the same authors again observed significant PE, even with time intervals of up to four years [21]. Given this finding, it is also likely that PE may affect whether one performs above or below a single cut-point and thus influence case ascertainment in longitudinal clinical trials.

In the present study, we sought to examine PE on the Memory Impairment Screen (MIS) over four annual administrations. Brief memory screening instruments are often used in clinical practice and research to identify those patients who might benefit from a more extensive clinical assessment, and whether specific individuals should be included in a research study. Some studies, such as the PREADViSE trial, rely on dementia screening measures to determine whether a participant should be evaluated with more in-depth cognitive assessment. More specifically, if performance on screening measures is influenced by PE, participants who may be cognitively impaired or demented will be adjudicated as cognitively normal and thus misclassified or potentially lost to follow-up. Given previous data on short-term and longterm PE, we hypothesized that despite efforts to mitigate $\mathrm{PE}$ through alternate test versions, MIS scores would improve over time.

\section{Methods}

\section{Participants}

For details on recruitment and design of the National Institutes of Health (NIH) National Institute on Agingsponsored PREADViSE trial, please see Kryscio et al. [4]. Briefly, the primary aim is to determine the effectiveness of the antioxidant supplements vitamin $\mathrm{E}$ and selenium in preventing the onset of AD. The PREADViSE trial recruited a subsample $(n=7,547)$ of participants age 62 and over (age 60 if of African-American descent) from the NIH National Cancer Institute-sponsored Selenium and Vitamin E Cancer Prevention Trial (SELECT) from 130 participating clinical sites in the US, Canada, and Puerto Rico. Men enrolled in both the SELECT prostate cancer study [27] and the PREADViSE trial, who completed baseline and three consecutive follow-up assessments, and obtained less than a perfect score at one or more assessments $(n=1,803)$ were included in the current analyses. Men with four consecutive assessments were selected to provide adequate follow-up to examine potential $\mathrm{PE}$, and men with perfect scores at all assessments were excluded because their scores could not improve. However, these men $(n=1,291)$ were included in a sensitivity analysis.

Despite bi-annual training sessions on the screening protocol with the site clinical research assistants (CRAs) [28], there were administration errors that resulted in some men receiving the same MIS version at consecutive visits. Thus, an additional subset of men who received the same version of the test protocol (due to CRA error) at baseline and first follow-up and obtained less than a perfect score at any of the four assessments $(n=301)$ were also analyzed to determine the effectiveness of alternate forms in mitigating PE.

This study was approved by the University of Kentucky Institutional Review Board as well as the Institutional Review Boards at all participating centers.

\section{Clinical evaluation}

The study employs a two-tier cognitive screening procedure for identification of memory impairment and dementia. The first consists of the MIS [29], which is administered at each annual visit. Participants who score below the predetermined cut-point on the MIS undergo a more extensive cognitive evaluation and medical workup [28]. The MIS was chosen for its brevity (under five minutes) and ease of administration with minimal 
training by CRAs, who were well-versed in cancer research but had no other training or experience in administering cognitive tests. To minimize PE, the alternate form of the MIS [29] was also included in the protocol for subsequent annual assessments. At each follow-up screen, the participant received the version not administered to him the previous year. During MIS administration, the participant is shown four written words and verbally given a category cue for each; after a 2-minute interval filled with a non-memory-based distraction task, the participant is asked to recall the words (free recall). Category cues may be given as needed to stimulate recall (cued recall). Two points are awarded for each correct free recall word, and one point is scored for each correct word following category cue. MIS total scores range from 0 to 8 points with 8 points indicating a perfect score, and a standard cut-point, recommended by Buschke and the test authors [29] is a score of 4. MIS screening began in May 2002 and will continue through January 2013. The cut-point was raised to 5 in January 2009 to capture participants potentially functioning in the $\mathrm{MCI}$ range.

\section{Statistical methods}

Linear mixed models (LMM) were used to test the hypothesis that MIS scores improve over time due to PE. Random intercepts and an unstructured covariance matrix were used to account for within-subject correlation. Initial models included fixed effects for age at baseline (centered at 70), education level (high school or lower, college or higher), race (African-American vs. not African-American), MIS version (version 1 vs. version 2 ), and annual visit, which was treated as acategorical variable. Two-way interactions between visit and age, race, and education were then added to the model.

Standard two-group comparisons (for example, $t$-tests and chi-square $\left(\chi^{2}\right)$ tests) were used to assess comparability between the men who received alternating versions over four visits and those who received the same version at baseline and first follow-up. Statistical significance was set at $\alpha=0.05$. All analyses were performed with SAS/STAT $9.3^{\circledR}$ software.

\section{Results}

Participants had an average \pm SD baseline age of $68.0 \pm$ 5.3 years and were highly educated, with $75.6 \%$ percent reporting at least some college education (Table 1). Participants with alternating MIS versions had received similar levels of education to those given the same version at baseline and follow-up but were slightly younger $(t=-2.18$, degrees of freedom $(\mathrm{df})=380.88, P=0.03)$ and comprised fewer minorities $\left(\chi^{2}=33.4 \mathrm{df}=2, P<\right.$ 0.0001). Almost half the men (47.0\%) who received alternating versions obtained a perfect score at baseline
Table 1 Baseline participant characteristics

\begin{tabular}{llll}
\hline Characteristics & Alternating versions & & \multicolumn{1}{c}{ Same version } \\
\cline { 2 - 2 } Age, years, mean (SD) & $67.8(5.2)$ & & $\mathbf{( n = 3 0 1 )}$ \\
Race, $\mathbf{n}$ (\%) & & $68.6(5.9)$ \\
African-American & $133(7.4)$ & \\
White & $1629(90.4)$ & & $27(9.0)$ \\
Other & $41(2.3)$ & $249(82.7)$ \\
Education level, $\mathbf{n}(\%)$ & & $25(8.3)$ \\
Unknown & $8(0.4)$ & \\
High school or lower & $447(24.8)$ & $0(0.0)$ \\
Some college or higher & $1348(74.8)$ & $63(20.9)$ \\
\hline
\end{tabular}

(Table 2), and about half (49.8\%) of those maintained their perfect score at the first follow-up screen (data not shown). Among men who did not achieve a perfect baseline score $(\mathrm{n}=955), 70.5 \%$ improved their score at the first follow-up screen while just $6.0 \%$ performed worse. Between baseline and follow-up visit 3, a 16.2\% increase in the proportion of men obtaining a perfect score was observed along with corresponding decreases in the proportion of men obtaining less than perfect scores (McNemar's $X^{2}=5.5, \mathrm{df}=1, P=0.02$ ) (Table 2). By

Table 2 Memory Impairment Screen (MIS) scores by visit

\begin{tabular}{|c|c|c|c|c|}
\hline \multicolumn{5}{|c|}{ All participants with alternating versions $(n=1,803)$} \\
\hline & $\begin{array}{l}\text { Baseline } \\
\text { MIS }\end{array}$ & FU 1 MIS & FU 2 MIS & FU 3 MIS \\
\hline $\begin{array}{l}\text { MIS score, mean } \\
\text { (SD) }\end{array}$ & $7.34(0.74)$ & $7.47(0.68)$ & $7.48(0.70)$ & $7.54(0.69)$ \\
\hline \multicolumn{5}{|l|}{ MIS score, n (\%) } \\
\hline$\leq 5$ & $45(2.5 \%)$ & $24(1.3 \%)$ & $30(1.7 \%)$ & $33(1.8 \%)$ \\
\hline 6 & $151(8.4 \%)$ & $123(6.8 \%)$ & $128(7.1 \%)$ & $103(5.7 \%)$ \\
\hline 7 & $759(42.1 \%)$ & $632(35.1 \%)$ & $596(33.1 \%)$ & $527(29.2 \%)$ \\
\hline 8 & $848(47.0 \%)$ & $\begin{array}{l}1,024 \\
(56.8 \%)\end{array}$ & $\begin{array}{l}1,049 \\
(58.2 \%)\end{array}$ & $\begin{array}{l}1,140 \\
(63.2 \%)\end{array}$ \\
\hline Total & $\begin{array}{l}1,803 \\
(100 \%)\end{array}$ & $\begin{array}{l}1,803 \\
(100 \%)\end{array}$ & $\begin{array}{l}1,803 \\
(100 \%)\end{array}$ & $\begin{array}{l}1,803 \\
(100 \%)\end{array}$ \\
\hline
\end{tabular}

Participants age $\mathbf{7 5}$ years or more at baseline with alternating versions $(n=218)$

\begin{tabular}{lllll}
\hline & $\begin{array}{l}\text { Baseline } \\
\text { MIS }\end{array}$ & FU 1 MIS & FU 2 MIS & FU 3 MIS \\
\hline $\begin{array}{l}\text { MIS score, mean } \\
\text { (SD) }\end{array}$ & $7.30(0.81)$ & $7.41(0.71)$ & $7.33(0.76)$ & $7.32(0.82)$ \\
MIS score, n (\%) & & & & \\
$\leq 5$ & $8(3.7 \%)$ & $2(0.9 \%)$ & $5(2.3 \%)$ & $10(4.6 \%)$ \\
6 & $24(11.0 \%)$ & $22(10.1 \%)$ & $23(10.6 \%)$ & $19(8.7 \%)$ \\
7 & $80(36.7 \%)$ & $79(36.2 \%)$ & $84(38.5 \%)$ & $80(36.7 \%)$ \\
8 & $106(48.6 \%)$ & $115(52.8 \%)$ & $106(48.6 \%)$ & $109(50.0 \%)$ \\
Total & $218(100 \%)$ & $218(100 \%)$ & $218(100 \%)$ & $218(100 \%)$ \\
\hline
\end{tabular}

FU, follow-up visit. 
contrast, although the same proportion of men $(47.0 \%)$ made a perfect score at baseline, a $14.0 \%$ increase in perfect scores was observed between baseline and follow-up visit 1 among the men who received the same version at the two assessments (McNemar's $\chi^{2}=10.9$, df $=1, P=$ 0.001) (data not shown).

For the men with four visits and alternating MIS versions, all main effects except race were significant in the initial LMM. Two-way interactions of age, education, and version with visit were significant when added to this model. A three-way interaction among age, education, and visit was also tested and was not significant. Hence, the final LMM contained random intercepts (that is, subject effects); main effects for age, education, visit, and MIS version; and visit by age, visit by education, and visit by version interaction terms. The effects of age and education in this model are illustrated in Table 3. While there were few significant PE at the first follow-up, which is unremarkable given almost half the men already had a perfect score at baseline, the youngest men (age 60 years) and men with the highest educational level did show a significant increase over baseline and maintained this PE through the third follow-up. By the second follow-up assessment a significant increase over baseline, which was maintained at the third followup, was observed for both educational levels and for all men age 70 years or younger. Although their mean scores did not decrease monotonically, men age 75 years and older showed few significant PE and tended to have lower estimated scores over time. Only the estimated mean scores at follow-up visit 2 for men age 75 years were significantly higher than baseline, while by followup visit 3 men age 85 years had estimated mean scores significantly lower than baseline. Finally, between baseline and follow-up visit 3 , the proportion of men age 75 years and older at baseline $(n=218)$ who achieved a perfect score increased by just $1.4 \%$ in contrast to $70.5 \%$ for the entire sample receiving alternate forms (Table 2).

Results changed little when men who obtained a perfect score at all four assessments were included in the analysis. While there were no significant PE at follow-up visit 1 for any age or educational level, PE were first observed among the youngest and best educated participants, while the oldest participants (age 80 years or more) had significantly lower estimated mean MIS scores at the third follow-up than at baseline.

For the men who were given the same MIS version at baseline and first follow-up (Table 4), only main effects for age, education, version, and visit were significant predictors of MIS score. For these men, there was a significant improvement from baseline in the overall mean at the first follow-up $(0.20 \pm 0.05$ point increase, $P=0.0005)$, and this holds true for even the oldest participants $(0.17 \pm 0.07$ point increase, $P=0.02$ ). Further, at follow-up visit 2, where $75 \%$ of participants received the alternate version for the first time, there was a non-significant decrease in the overall mean $(0.10 \pm 0.06$ points, $P=0.098)$, all of which underscores the importance of alternating instrument versions to minimize PE in screening. These men were quite similar to those who received alternating versions of the MIS on all baseline characteristics except race, which is likely due to MIS administration errors occurring more often at certain study sites that happened to have more non-white participants. Since race was not a significant predictor in this analysis, however, the higher proportion of African-Americans and other races in this group does not explain the PE.

\section{Discussion}

Determining the success or failure of a dementia prevention trial depends heavily on the ability of the investigators to ascertain caseness. In a large trial, where budget

Table 3 Adjusted mean Memory Impairment Screen (MIS) scores based on a linear mixed model (LMM): alternating versions from baseline through follow-up (FU) visit 3 ( $n=1,803$ )

\begin{tabular}{lllll}
\hline LMM factor & Baseline MIS & FU 1 MIS & FU 2 MIS & FU 3 MIS \\
\hline $\begin{array}{l}\text { Education } \\
\quad \text { High school }\end{array}$ & $7.29(0.03)$ & $7.28(0.03)$ & & \\
$\quad$ Some college & $7.40(0.02)$ & $7.46(0.02)^{1}$ & $7.45(0.03)^{1,2}$ & $7.53(0.02)^{1,2}$ \\
Age, years & & & & $7.53(0.02)^{1,3}$ \\
60 & $7.37(0.03)$ & $7.46(0.03)^{1}$ & $7.61(0.03)^{1,2}$ & $7.71(0.03)^{1,2,3}$ \\
65 & $7.36(0.02)$ & $7.41(0.02)$ & $7.53(0.02)^{1,2}$ & $7.59(0.02)^{1,2}$ \\
70 & $7.34(0.02)$ & $7.35(0.02)$ & $7.46(0.02)^{1,2}$ & $7.48(0.02)^{1,2}$ \\
75 & $7.33(0.03)$ & $7.29(0.03)$ & $7.38(0.03)^{2}$ & $7.36(0.03)$ \\
80 & $7.31(0.04)$ & $7.23(0.04)$ & $7.31(0.04)$ & $7.25(0.04)$ \\
85 & $7.29(0.06)$ & $7.17(0.06)$ & $7.23(0.06)$ & $7.13(0.06)^{4}$ \\
\hline
\end{tabular}

Results are presented as mean score (SEM). ${ }^{1}$ significantly higher than baseline; ${ }^{2}$ significantly higher than FU $1 ;{ }^{3}$ significantly higher than FU $2 ;{ }^{4}$ significantly lower than baseline. 
Table 4 Adjusted mean Memory Impairment Screen (MIS) scores based on a linear mixed model (LMM): same version at baseline and follow-up (FU) visit $1(n=301)$

\begin{tabular}{lllll}
\hline LMM Factor & Baseline MIS & FU 1 MIS & FU 2 MIS & FU 3 MIS \\
\hline $\begin{array}{l}\text { Education } \\
\text { < High school }\end{array}$ & $7.25(0.05)$ & & & \\
$\quad$ Some college & $7.35(0.05)$ & $7.36(0.09)$ & $7.31(0.09)$ & $7.40(0.10)$ \\
Age, years & & $7.57(0.05)^{1}$ & $7.45(0.05)$ & $7.65(0.05)^{1,2}$ \\
60 & $7.48(0.07)$ & & & \\
65 & $7.38(0.06)$ & $7.65(0.07)^{1}$ & $7.57(0.07)$ & $7.71(0.07)^{1}$ \\
70 & $7.27(0.05)$ & $7.54(0.06)^{1}$ & $7.46(0.06)$ & $7.60(0.06)$ \\
75 & $7.16(0.06)$ & $7.43(0.05)^{1}$ & $7.35(0.06)$ & $7.49(0.05)^{1}$ \\
80 & $7.05(0.07)$ & $7.32(0.06)^{1}$ & $7.24(0.06)$ & $7.39(0.06)^{1}$ \\
85 & $6.94(0.09)$ & $7.22(0.07)^{1}$ & $7.14(0.07)$ & $7.28(0.07)^{1}$ \\
\hline
\end{tabular}

Results are presented as mean score (SEM). ${ }^{1}$ significantly higher than baseline; ${ }^{2}$ significantly higher than FU 2.

and time constraints may dictate the use of uncomplicated screening instruments, unrecognized PE may mask impairment and consequently bias results. In such cases it is desirable to minimize PE to identify individuals who need further evaluation.

We examined PE over four annual presentations of a brief memory screen, the MIS. In contrast to several previous studies of other instruments, we found a robust PE between the first and second presentation only when identical test forms were used. Use of alternate versions largely mitigated the PE at first follow-up, although PE was observed for those with at least some college education and for the youngest participants, which is consistent with findings from other studies. Interestingly, there were few PE for the oldest participants when alternate forms were used consistently. In fact, similar to the findings from the 5-year Personnes Agées QUID (PAQUID) study [30], these participants tended to do worse over time, which may support the hypothesis that a lack of PE may signal early cognitive decline $[8,31,32]$.

The study population consisted only of men, and therefore potential gender differences could not be studied, therefore the generalizability of findings to women is uncertain. Moreover, treatment effects of vitamin $\mathrm{E}$ and selenium, if they exist, could not be assessed as the investigators remain blinded to treatment arm. The utility of these results is limited by the nature of the MIS, an exclusively memory-based measure that neglects other areas of cognitive functioning. In addition, because of the restricted range and clear ceiling effect with this instrument, men who had a perfect score could not improve; the floor effect was not a factor since none of the participants in our study scored zero. However, the relatively small mean increases in scores between visits reflect the limited range of the MIS and should not be mistaken for clinically insignificant changes. It is notable that PE of any magnitude were found on a brief, four-item screening measure with identical versions being presented two years apart. Further, while the changes in the means were small, the proportion of perfect scores increased steadily and quite dramatically over time, if alternating versions were not used. These results continue to support the use of alternate forms in clinical and research settings where identifying candidates for further evaluation is the goal.

This study contributes to the literature in several ways. First, it adds to the information on the variability of cognitive screening measures across long periods of time, especially for longitudinal aging trials. It also adds to the information on the performance of the MIS as a brief screening measure for participants of varying age, education, and ethnic background. These data should further serve to inform the design and implementation of future dementia prevention studies.

Although there are several longitudinal studies investigating reliable change indices ( $\mathrm{RCI})$, we view this as an issue that is related to but separate from PE. More specifically, RCI allow one to control for the effect of practice in determining whether there has been a reliable change in cognition over time. Screening measures used in longitudinal studies are typically not used to detect subtle declines per se but rather to re-screen participants for inclusion into or exclusion from a study. Additionally, some studies have shown that RCIs must be rather large to reflect credible change [33-37]. However, a PE of just one point can be consequential enough to have detrimental effects on case ascertainment.

\section{Conclusion}

In this paper, we present the results of PE on a sample of 2,104 cognitively intact adult men over age 60 years, tested annually over four years. Strengths of the design itself include the large sample size, longitudinal nature of the study, and use of alternate forms for the vast majority of examinees. This study also demonstrates subtle 
but important shifts toward improved scores over time on a brief screening measure. Given the importance of repeated brief screening measures to clinical trial case ascertainment, our study highlights the importance of evaluating the effect of practice on specific instruments used in longitudinal clinical trials. Future research may wish to explore the possibility of adjusting cut-points on repeated measures, and determining the effect this might have on overall case ascertainment.

\section{Competing interests}

The authors declare that they have no competing interests.

\section{Authors' contributions}

ELA prepared the data, performed all statistical analyses, helped draft the manuscript, and revised this manuscript for resubmission to Trials. BCD helped draft the manuscript. MJM helped draft and revise the manuscript. MSM participated in the design of the study and helped draft the manuscript. ACH trained site staff on the screening protocol and participated in study coordination. RJK participated in the design of the study, oversaw the statistical analysis plan, and helped draft the manuscript. FAS participated in the design and coordination of the study, trained site staff on the screening protocol, and helped draft the manuscript. JCJ participated in the design of the study and helped coordinate data collection. All authors read and approved the final manuscript.

\section{Acknowledgments}

The PREADViSE trial (NCT 00040378) is supported by grant R01 AG019241 from the $\mathrm{NIH}$ - National Institute on Aging, Bethesda, Maryland, USA. The SELECT trial (NCT 00076128) is supported by the NIH - National Cancer Institute, Bethesda, Maryland, USA.

\section{Author details}

${ }^{1}$ Sanders-Brown Center on Aging, University of Kentucky, Lexington, KY, USA. ${ }^{2}$ Departments of Neurology, University of Kentucky Lexington, Kentucky, USA. ${ }^{3}$ Departments of Biostatistics, University of Kentucky, Lexington, KY, USA. ${ }^{4}$ Departments of Statistics, University of Kentucky, Lexington, KY, USA. ${ }^{5}$ Departments of Behavioral Science, University of Kentucky, Lexington, KY, USA. ${ }^{6}$ Departments of Psychiatry, University of Kentucky, Lexington, KY, USA. ${ }^{7}$ Cancer Research and Biostatistics, Seattle, WA, USA. ${ }^{8}$ Sanders-Brown Center on Aging, University of Kentucky College of Medicine, Lexington, KY, USA.

Received: 28 February 2012 Accepted: 10 October 2012

Published: 20 November 2012

\section{References}

1. Lezak MD, Howieson DB, Loring DW, Hannay HJ, Fischer JS: Neuropsychological assessment. 4th edition. New York: Oxford University Press; 2004.

2. Meinert $C L$, McCaffrey LD, Breitner JC: Alzheimer's disease antiinflammatory prevention trial: design, methods, and baseline results. Alz Dement 2009, 5:93-104.

3. DeKosky ST, Williamson JD, Fitzpatrick AL, Kronmal RA, Ives DG, Saxton JA, Lopez OL, Burke G, Carlson MC, Fried LP, Kuller LH, Robbins JA, Tracy RP, Woolard NF, Dunn L, Snitz BE, Nahin RL, Furberg CD, Ginkgo Evaluation of Memory (GEM) Study Investigators: Ginkgo biloba for prevention of dementia: a randomized controlled trial. JAMA 2008, 300:2253-2262.

4. Kryscio RJ, Mendiondo MS, Schmitt FA, Markesbery WR: Designing a large prevention trial: statistical issues. Stat Med 2004, 23:285-296.

5. Versavel MvL D, Evertz C, Unger S, Meier F, Kuhlman J: Test-retest reliability and influence of practice effects on performance in a multi-user computerized psychometric test system for use in clinical pharmacological studies. Drug Res 1997, 47:781-786.

6. Benedict RH, Zgaljardic DJ: Practice effects during repeated administrations of memory tests with and without alternate forms. J Clin Exp Neuropsychol 1998, 20:339-353.

7. Bird CM, Kyriaki P, Ricciardelli P, Rossor MN, Cipolotti L: Test-retest reliability, practice effects and reliable change indices for the recognition memory test. British J Clin Psychol 2003, 42:407-425.
8. Cooper DB, Lacritz LH, Weiner MF, Rosenberg RN, Cullum CM: Category fluency in mild cognitive impairment; reduced effect of prectice in testretest conditions. Alz Dis Assoc Disord 2004, 18:120-122.

9. Falleti MG, Maruff P, Collie A, Darby DG: Practice effects associated with the repeated assessment of cognitive function using the CogState battery at 10-minute, one week and one month test-retest intervals. J Clin Exp Neuropsychol 2006, 28:1095-1112.

10. Troster Al, Woods SP, Morgan EE: Assessing cognitive change in Parkinson's disease: development of practice effect-corrected reliable change indices. Arch Clin Neuropyschol 2007, 22:711-718.

11. Folstein MF, Folstein SE, McHugh PR: "Mini-mental state". A practical method for grading the cognitive state of patients for the clinician. J Psychiatr Res 1975, 12:189-198.

12. Galasko D, Abramson I, Corey-Bloom J, Thal LJ: Repeated exposure to the mini-mental state examination and the information memoryconcentration test results in a practice effect in Alzheimer's disease. Neurology 1993, 43:1559-1563.

13. Watson FL, Pasteur ML, Healy DT, Hughes EA: Nine parallel versions of four memory tests: an assessment of form equivalence and the effects of practice on performance. Human Psychopharmacol Clin Exp 1994, 9:51-61.

14. Basso MR, Carona FD, Lowery N, Axelrod BN: Practice effects on the WAISIII across 3- and 6-month intervals. Clin Neuropsychol 2002, 16:57-63.

15. Duff K, Westervelt HJ, McCaffrey RJ, Haase RF: Practice effects, test-retest stability, and dual baseline assessments with the California verbal learning test in an HIV sample. Arch Clin Neuropsychol 2001, 16:461-476.

16. Johnson BF, Hoch K, Johnson J: Variability in psychometric test scores: the importance of the practice effect in patient study design. Prog Neuropsychopharmacol Biol Psychiatry 1991, 15:625-635.

17. McCaffrey RJ, Ortega A, Orsillo SM, Nelles WB: Practice effects in repeated neuropsychological assessments. Clin Neuropsychol 1992, 6:32-42.

18. Mitrushina M, Satz P: Effect of repeated administration of a neuropsychological battery in the elderly. J Clin Psychol 1991, 47:790-801.

19. Rabbitt P, Banerji N, Szymanski A: Space Fortress as an IQ test? Predictions of learning and of practised performance in a complex interactive videogame. Acta Psychol 1989, 71:243-257.

20. Rabbitt P, Diggle P, Holland F, Mclnnes L: Practice and drop-out effects during a 17-year longitudinal study of cognitive aging. J Gerontol B Psychol Sci Soc Sci 2004, 59:P84-P97.

21. Rabbitt $P$, Lunn M, Wong D, Cobain M: Age and ability affect practice gains in longitudinal studies of cognitive change. J Gerontol B Psychol Sci Soc Sci 2008, 63:P235-P240.

22. Heaton RK, Temkin N, Dikmen S, Avitable N, Taylor MJ, Marcotte TD, Grant I: Detecting change: a comparison of three neuropsychological methods, using normal and clinical samples. Arch Clin Neuropsychol 2001, 16:75-91.

23. Dikmen SS, Heaton RK, Grant I, Temkin NR: Test-retest reliability and practice effects of expanded halstead-reitan neuropsychological test battery. J Int Neuropsychol Soc 1999, 5:346-356.

24. McCaffrey RJ, Ortega A, Haase RF: Effects of repeated neuropsychological assessments. Arch Clin Neuropsychol 1993, 8:519-524.

25. Salinsky MC, Storzbach D, Dodrill CB, Binder LM: Test-retest bias, reliability, and regression equations for neuropsychological measures repeated over a 12-16-week period. J Int Neuropsychol Soc 2001, 7:597-605.

26. Duff K, Beglinger $\amalg$, Schultz SK, Moser DJ, McCaffrey RJ, Haase RF, Westervelt HJ, Langbehn DR, Paulsen JS, Huntington's Study Group: Practice effects in the prediction of long-term cognitive outcome in three patient samples: a novel prognostic index. Arch Clin Neuropsychol 2007, 22:15-24.

27. Lippman SM: Designing the selenium and vitamin E cancer prevention trial (SELECT). J Natl Cancer Inst 2005, 97:94-102.

28. Kryscio RJ, Abner EL, Schmitt FA, Goodman PJ, Mendiondo M, Caban-Holt A, Dennis BC, Mathews M, Klein EA, Crowley JJ: A randomized controlled Alzheimer's disease prevention trial's evolution into an exposure trial: The PREADVISE trial., . in press.

29. Buschke H, Kuslansky G, Katz M, Stewart WF, Sliwinski MJ, Eckholdt HM, Lipton RB: Screening for dementia with the memory impairment screen. Neurology 1999, 52:231-238.

30. Jacamin-Gadda H, Fabrigoule C, Commenges D, Dartigues JF: A 5-year longitudinal study of the mini-mental state examination in normal aging. Am J Epidemiol 1997, 145:498-506.

31. Duff K, Lyketsos CG, Beglinger LJ, Chelune G, Moser DJ, Arndt S, Schultz SK Paulsen JS, Petersen RC, McCaffrey RJ: Practice effects predict cognitive 
outcome in amnestic mild cognitive impairment. Am J Geriatric Psych 2011, 19:932-939.

32. Galvin JE, Powlishta KK, Wilkins K, McKeel DW Jr, Xiong C, Grant E, Storandt $M$, Morris JC: Predictors of preclinical Alzheimer disease and dementia: a clinicopathologic study. Arch Neurol 2005, 62:758-765.

33. Bird CM, Papadopadopoulou K, Ricciardelli P, Rossor MN, Cipolotti L: Testretest reliability, practice effects and reliable change indices for the recognition memory test. Brit J Clin Psychol 2003, 42:407-425.

34. Chelune GJ, Naugle RI, Luders H, Sedlak J, Awad IA: Individual change after epilepsy surgery: practice effects and base-rate information. Neuropsychol 1993, 7:41-52

35. Sachs BC, Lucas JA, Smith GE, Ivnik RJ, Petersen RC, Graff-Radford NR,

Pedraza O: Reliable change on the Boston naming test. J Int Neuropsychol Soc 2012, 18:375-378.

36. Pedraza O, Smith GE, Ivnik RJ, Willis FB, Ferman TJ, Petersen RC, GraffRadford NR, Lucas JA: Reliable change on the dementia rating scale. J Int Neuropsychol Soc 2007, 13:716-720

37. Ivnik RJ, Smith GE, Lucas JA, Petersen RC, Boeve BF, Kokmen E, Tangalos EG: Testing normal older people three or four times at 1- to 2-year intervals: defining normal variance. Neuropsychol 1999, 13:121-127.

doi:10.1186/1745-6215-13-217

Cite this article as: Abner et al:: Practice effects in a longitudinal, multicenter Alzheimer's disease prevention clinical trial. Trials 2012 13:217.

\section{Submit your next manuscript to BioMed Central and take full advantage of:}

- Convenient online submission

- Thorough peer review

- No space constraints or color figure charges

- Immediate publication on acceptance

- Inclusion in PubMed, CAS, Scopus and Google Scholar

- Research which is freely available for redistribution 